\title{
Selected Reaction Monitoring
}

National Cancer Institute

\section{Source}

National Cancer Institute. Selected Reaction Monitoring. NCI Thesaurus. Code C161787.

A method for collecting tandem mass spectrometry data where an ion of a particular mass is selected in the first stage and a fragmented ion product is selected in the second mass spectrometer stage for detection. Selected reaction monitoring (SRM)-based experiments are generally performed in a triple quadrupole (QqQ) mass spectrometer. 